\title{
MODELING FLUID FLOWS IN DISTENSIBLE TUBES FOR APPLICATIONS IN HEMODYNAMICS
}

\author{
X. DESCOVICH*, G. PONTRELLI ${ }^{\dagger}$,, S. MELCHIONNA \\ S. SUCCI ${ }^{\dagger}$ and S. WASSERTHEURER $\$$ \\ *Institute of Analysis and Scientific Computing \\ Vienna University of Technology, Wieder Hauptstrasse 8-10 \\ 1040 Wien, Austria \\ ${ }^{\dagger} I A C$-CNR, Istituto per le Applicazioni del Calcolo \\ Via dei Taurini 19 - 00185 Roma, Italy \\ \giuseppe.pontrelli@gmail.com \\ IPCF-CNR, Istituto Processi Chimico-Fisici \\ Department of Physics, University La Sapienza \\ P. le A. Moro 2 - 00185 Roma, Italy \\ $\S_{A I T}$ Austrian Institute of Technology GmbH \\ Donau-City-Strasse 1, 1220 Wien, Austria
}

Received 16 January 2013

Accepted 28 February 2013

Published 25 April 2013

\begin{abstract}
We present a lattice Boltzmann (LB) model for the simulation of hemodynamic flows in the presence of compliant walls. The new scheme is based on the use of a continuous bounce-back boundary condition, as combined with a dynamic constitutive relation between the flow pressure at the wall and the resulting wall deformation. The method is demonstrated for the case of two-dimensional (axisymmetric) pulsatile flows, showing clear evidence of elastic wave propagation of the wall perturbation in response to the fluid pressure. The extension of the present two-dimensional axisymmetric formulation to more general three-dimensional geometries is currently under investigation.
\end{abstract}

Keywords: Lattice Bottzmann method; elastic walls; hemodynamics; stents.

PACS Nos.: 47.63.Cb, 87.10, 87.19.

\section{Introduction}

Cardiovascular diseases are the most common cause of death in the European Union $^{1}$ and in the industrialized world in general. There is considerable evidence that the development of such diseases is, to a great extent, linked to the characteristics of the blood flow and, in particular, to the arterial wall stiffening. ${ }^{2}$ Since, experimental methods in the cardiovascular system are difficult and invasive,

ๆ Corresponding author. 
mathematical models and numerical methods to simulate the hemodynamic processes have gained a growing importance over the years. ${ }^{3-6}$

Research in the field includes studies incorporating the whole arterial tree ${ }^{7}$ as well as studies of only parts of it, e.g. a segment of an artery. ${ }^{8}$ This paper is aimed at developing a simple method for the simulation of blood flow in a vessel segment and hence focuses on the investigation of local flow behavior.

Many applications of fluid dynamics deal with flows confined by rigid boundaries, such as flows in ducts and pipes. In some other applications, for instance hemodynamics, it is important to include the effects of the wall compliance. Since arteries are elastic and change in diameter depending on the pulsatile blood pressure inside, it is crucial to include elasticity effects in models of physiological flows in blood vessels. This means that appropriate models describing the dynamic fluid-structure interaction have to be developed. This is one of the major computational challenges in biomechanics. Many papers in the literature deal with fluid-structure interaction problems. ${ }^{9-11}$ They differ by the method by which the set of equations for the fluid component (e.g. Navier-Stokes equations) and those for the solid one (e.g. wall motion equations) are solved. A variety of coupling algorithms have been developed, such as the coupled and the weakly coupled approach, or the uncoupled method where the equations for the fluid and for the structure are computed separately. A short description of these algorithms can be found in Refs. 12-14.

Some of the above works dealing with fluid-structure interaction problems use independent grids for the fluid part and the solid part. This requires appropriate mappings between the variables describing the two components. A commonly used approach is the Arbitrary Lagrangian Eulerian (ALE) method, which provides a mapping between the Lagrangian system (in which the structure is commonly described) and the Eulerian system (in which the fluid is generally described). ${ }^{3}$ In the ALE approach, the physical boundary is moving, while the fictitious boundary (e.g. inlet/outlet section of a blood vessel) is kept fixed and undeformed. In such a technique, the grid is moving, which typical calls for re-meshing procedures in order to avoid extreme deformations which would endanger the stability of the simulation. An alternative method based on a nonmoving grid is the Cartesian cut cells approach. ${ }^{13}$ In it, no remeshing is needed, and the wall enclosing the fluid domain moves over a fixed grid.

The immersed fibers method proposed by Peskin in 1977 (Ref. 15) can be regarded as the first attempt to study fluid-structure interaction problems in the cardiovascular system. Since then, other methods have been developed. Conventional numerical schemes for fluid-structure interaction problems couple the finite element method for solving the governing equations of the structure, with a finite difference, finite element or finite volume method for the fluid description. The solid and fluid subsystems can be solved simultaneously ${ }^{16}$ or separately. ${ }^{17}$

A recent option, which offers a competitive alternative to the aforementioned numerical methods, especially on parallel computing architectures, is provided by the 
lattice Boltzmann (LB) method. ${ }^{18,19}$ This method has been applied to a broad variety of complex flows, ${ }^{20}$ including fluid-structure interactions problems..$^{9,10,12,14,21-23}$

Within the LB framework, the method proposed by Fang et al. ${ }^{21,22}$ provides conditions for elastic and moving boundaries. In this approach, virtual distribution functions at the boundary are introduced. The velocity at boundary nodes required to compute these virtual distribution functions is obtained by quadratic interpolation or extrapolation. This method has been successfully applied to LB simulations in two-dimensional elastic tubes by Hoekstra et al. ${ }^{23}$ It is accurate, but not appropriate for large simulations running in parallel because of the quadratic extrapolations and the fact that nodes change from the solid domain to the fluid domain and vice versa. ${ }^{10}$ Another modification of the LB scheme to model coupled fluidstructure problems has been proposed by Krafczyk et al. ${ }^{14} \mathrm{It}$ is based on the work of Ladd, who presented a general technique for the simulation of fluid-particle interactions. ${ }^{24}$ The method is a combination of the LB equation for the fluid domain and Newtonian dynamics of the solid particles. It allows to determine the interaction between fluid and solid boundary by directly using the LB variables. Krafczyk et al. applied the method to study the fluid-structure system of moving leaflets of an artificial heart valve driven by physiological blood flow. ${ }^{14}$ Other numerical simulations using the method of Ladd have been carried out. ${ }^{25,26}$ Chopard and Marconi ${ }^{9}$ modeled the fluid-wall interaction based on local exchange of momentum between solid and fluid particles. Their method differs from that in Ref. 24, in which the solid particles immersed in the fluid are rigid, and allows to model solid, deformable particles suspended in a fluid by using the LB method, for both the fluid and the solid phase. An approach coupling the LB model (for the fluid) with a lattice spring model (for the compliant wall) has been proposed by Buxton et al. ${ }^{12}$ A network of "springs" which are connected to each other describes the interaction. A major advantage of a lattice spring model is its computational efficiency. ${ }^{12}$ Efficient computing is also provided by the approach of Doctors et al. ${ }^{10}$ who performed LB simulations of pulsatile fluid flow in three-dimensional elastic pipes. Their method is "based on estimating the distances from sites at the edge of the simulation box to the wall along the lattice directions from the displacement of the closest point on the wall and the curvature there, followed by application of a nonequilibrium extrapolation method." 10

Quite recently, Leitner ${ }^{8}$ proposed a simple method for modeling elastic vessel walls in LB simulations of arterial blood flow. The method is similar to the Cartesian cut cells approach mentioned above. The wall displacement is based on the local pressure and involves transmural pressure corresponding to the pressure needed to balance the restoring forces from the elastic wall and to maintain this wall in equilibrium. The method presented by Leitner does not treat a realistic fluid-structure interaction, since it does not include the feedback from the wall to the fluid. Nevertheless, it provides satisfactory results for simulations of blood flow in artery segments, by keeping the numerical algorithm simple and robust. Inevitably, the method presents some drawbacks, for instance the fact that the wall cannot be 
displaced by less than one single lattice unit of the underlying grid. This stepwise wall displacement is a source of significant discretization errors, which may compromise the accuracy of the simulation.

This paper presents a simple and accurate method for incompressible flow through distensible tubes. Most of the material included here has been developed in an extended form in Ref. 27, and the reader is referred to it for more details. The present work introduces a novel boundary condition allowing a continuous displacement of the wall, as opposed to the aforementioned step-wise motion. The modeling of the elastic wall is kept fully local, hence basically as simple as Leitner's method, thereby preserving the efficiency of the LB algorithm.

A significant advantage of the present approach is that it can also be used for simulating the blood flow through stents, i.e. wire metal meshes inserted into a narrowed artery to prevent its occlusion. ${ }^{28}$ Due to the geometry and the different stiffness properties of the stent, separation and local recirculation can occur near the stented region. It is well recognized that such a recirculation may cause a re-narrowing of the artery, the so-called in-stent restenosis, which is a pathological process often occurring after stent implantation. ${ }^{29}$ Efficient methods to simulate the blood flow through stents are therefore of particular interest, to gain a deeper understanding of the physiological features of stented arteries.

\section{The LB Method for Fluid Flows}

The LB method is a mesoscopic approach to solve the Navier-Stokes equations starting from a minimal form of the Boltzmann kinetic equation. It describes the evolution of fictitious lumps of particles on nodes of a regular lattice with spacing $\Delta x$. The main variables are the distribution functions $f_{i}\left(\mathbf{x}, \mathbf{c}_{i}, t\right)$ (also called populations) which denote the probability of finding at time $t$ a particle located at site $\mathbf{x}$ and traveling along the lattice links in direction $i$, with the speed $\mathbf{c}_{i}$. Starting from an initial state, the populations evolve at every time-step through two consecutive substeps: (a) collision, describing the interaction of particles at a node, and (b) streaming, in which each particle is propagated to the neighboring node, based on the direction of its discrete velocity $\mathbf{c}_{i}$. A more detailed introduction to the LB method can be found in Refs. 18, 30 and 31.

The LB method is a valuable alternative to numerical methods based on the solution of the Navier-Stokes equations of continuum mechanics. Many authors have successfully and extensively applied LB models in hemodynamics. ${ }^{22,32-34}$

We assume here the flow to be Newtonian and use a D2Q9 model (two spatial dimensions, nine discrete speeds; see Fig. 1). The dynamics of the flow field is computed on the basis of the LB equation, with single-time relaxation collision operator:

$$
f_{i}\left(\mathbf{x}+\mathbf{c}_{i} \Delta t, t+\Delta t\right)-f_{i}(\mathbf{x}, t)=-\omega \Delta t\left(f_{i}-f_{i}^{\mathrm{eq}}\right)(\mathbf{x}, t),
$$

with $\Delta t$ being the time-step, taken equal to one in lattice units and $\omega$ the relaxation frequency. The right-hand side of Eq. (2.1) represents molecular collisions through a 


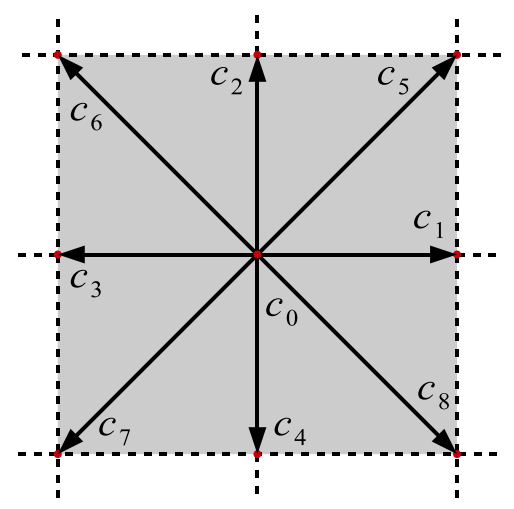

Fig. 1. (Color online) D2Q9 lattice.

relaxation towards a local equilibrium $f_{i}^{\text {eq }}$, which is given by a truncated Maxwell-Boltzmann distribution and expressed as:

$$
f_{i}^{\mathrm{eq}}=w_{i} \rho\left(1+3 \mathbf{c}_{i} \cdot \mathbf{u}+\frac{9}{2}\left(\mathbf{u} \cdot \mathbf{c}_{i}\right)^{2}-\frac{3}{2} \mathbf{u}^{2}\right),
$$

with $w_{i}$ being a set of weights associated with the discrete velocities $\mathbf{c}_{i}$. The fluid density $\rho$ and the momentum $\rho \mathbf{u}$ are obtained from the population $f_{i}$ by suitable summations upon all possible directions:

$$
\begin{gathered}
\rho(\mathbf{x}, t)=\sum_{i} f_{i}(\mathbf{x}, t), \\
\rho(\mathbf{x}, t) \mathbf{u}(\mathbf{x}, t)=\sum_{i} \mathbf{c}_{i} f_{i}(\mathbf{x}, t),
\end{gathered}
$$

where $\mathbf{c}_{i}$ is a set of discrete velocities connecting a mesh node to the mesh neighbors and a null vector for the case $i=0$ (rest particle).

A crucial aspect of hemodynamics in compliant vessels regards the modeling and control of the propagation of pressure waves traveling along the system. It is well known that two ingredients determine the speed and dispersion of a traveling wave, the wall distensibility and the fluid density $\rho .{ }^{35}$ To appreciate this, let us consider the ideal case of an incompressible inviscid fluid in a distensible tube, subject to a small pressure perturbation. The $1 \mathrm{D}$ continuity and momentum equation reads as follows:

$$
\begin{gathered}
\frac{\partial A}{\partial t}+\frac{\partial(A u)}{\partial x}=0 \\
\frac{\partial u}{\partial t}+u \frac{\partial u}{\partial x}+\frac{1}{\rho} \frac{\partial p}{\partial x}=0
\end{gathered}
$$

where $A$ denotes the cross-sectional area, $u$ the cross-section averaged longitudinal velocity and $p$ the pressure. By supposing a circular cross-sectional area $A=\pi R^{2}$ and linearizing the previous equations, the combination of Eqs. (2.5) and (2.6) provides 
the wave equation for $R(x, t)$ :

$$
\frac{\partial^{2} R}{\partial t^{2}}-c_{w}^{2} \frac{\partial^{2} R}{\partial x^{2}}=0
$$

with the associated wave speed $c_{w}=\sqrt{\frac{1}{\rho \beta}}$, being $\beta=\frac{1}{A} \frac{d A}{d p}$ the wall distensibility, which involves both wall and fluid properties. Physiological values are typically $c_{w} \sim 5-10 \mathrm{~m} / \mathrm{s}^{35}$ By the further assumption of a thin wall (with wall thickness $h$ ) that behaves as a linearly elastic isotropic Hookean body (with Young modulus $E$ ), the Moens-Korteweg formula $c_{w}=\sqrt{\frac{E h}{2 \rho R}}$ is easily derived. ${ }^{35}$ In real cases, however, deviations from ideality stem from the fluid viscosity and the wall viscoelasticity and, as a result of these viscous losses, the wall oscillations are damped.

Given the above discussion, in our treatment we model the deformation of the elastic wave explicitly, with a velocity that depends on the local fluid density. The fluid response to compression couples with the wall deformation via a constitutive relation for the transmural pressure, specified later on, and with the propagation speed of the elastic wave. In LB practical implementations, however, we consider the fluid as compressible and viscous. Moreover, it is important to control the speed of propagation of the sound wave, in order to avoid high-frequency sonic reflections at the outlet, that eventually destabilize the numerical scheme. To this purpose, we introduce a body force on the R.H.S. of Eq. (2.1) which produces a variable speed of sound ${ }^{36,37}$ :

$$
f_{i}\left(\mathbf{x}+\mathbf{c}_{i} \Delta t, t+\Delta t\right)-f_{i}(\mathbf{x}, t)=-\omega \Delta t\left(f_{i}-f_{i}^{\mathrm{eq}}\right)(\mathbf{x}, t)+3 w_{i} \gamma \nabla \rho \cdot \mathbf{c}_{i} .
$$

As a matter of fact, the tunable parameter $\gamma$ in the forcing term of Eq. (2.8) controls the fluid sound speed $c_{s}=\sqrt{\frac{1}{3}-\gamma}$. For example, $c_{s} \in[0,2 / \sqrt{3}]$ for $\gamma \in[-1,1 / 3]$. By the Chapman-Enskog asymptotic expansion, the Navier-Stokes equations for a weakly compressible fluid are derived:

$$
\begin{gathered}
\frac{1}{c_{s}^{2}} \frac{\partial p}{\partial t}+\nabla \cdot \mathbf{u}=0 \\
\frac{\partial \mathbf{u}}{\partial t}+\mathbf{u} \nabla \cdot \mathbf{u}=-\frac{1}{\rho} \nabla p+\nu \nabla^{2} \mathbf{u}
\end{gathered}
$$

with the equation of state $p=c_{s}^{2} \rho$ and with $\nu=\frac{2-\omega}{6 \omega}$ the kinematic viscosity. The limit of quasi-inviscid fluid is attained by choosing $\omega \simeq 2$. The term weakly compressible means that the fluid supports local density changes of the order of $\delta \rho / \rho \sim M a^{2}, M a=$ $u / c_{s}$ being the Mach number of the flow.

Inlet and outlet fluid boundaries impose a fixed pressure, according to the popular Zou-He procedure. ${ }^{38}$ The procedure prescribes the off-equilibrium components of the incoming population according to the following relation:

$$
f_{i}-f_{i}^{\mathrm{eq}}=f_{\bar{i}}-f_{\bar{i}}^{\mathrm{eq}},
$$

where $\bar{i}$ refers to the opposite/conjugate discrete directions $\left(\mathbf{c}_{i}=-\mathbf{c}_{i}\right)$. Being explicit, such scheme is particularly simple to implement and robust for small and intermediate Reynolds numbers. Full details are found in Ref. 38. 


\section{Modeling the Moving Wall}

We have developed a simple approach to model the elastic vessel that keeps the parametrization of the wall to a bare minimum. The modeling is inspired by the work of Leitner ${ }^{8}$ and acts strictly locally, in the spirit of the LB method. In this way, the complexity of the algorithm is kept at a manageable level.

In a 2D Cartesian reference system, let us consider a rectangular domain of dimensions $N_{x} \times N_{y}$, where $N_{x}$ and $N_{y}$ are the number of lattice nodes in direction $x$ and $y$, respectively. In the modeling of the vessel wall, the nodes of the lattice can have two different states: fluid, representing the blood flowing in the vessel, and solid, denoting the tissue of the vessel. Differently than in Ref. 8, the vessel, wall is generally not located on the solid nodes but in between fluid and solid nodes. All nodes that are not fluid are by default solid ones. The approach does not require cellular automata as used in Leitner's method to avoid the rupture of the vessel wall. The displacement of the wall is modeled by changing the type of a node - from solid to fluid for an expansion and vice versa for an inwards displacement for a contraction. The change of the node type is dependent on the local pressure of the surrounding fluid nodes. Figure 2 illustrates the concept of wall displacement based on node type changes.

The presented method is an implementation of the hemoelastic feedback system described by Fung ${ }^{39}$ which consists of two functional units: an elastic body and a pressure induced fluid mechanism. The vessel is considered as a rigid fluid conduit having a certain shape. The pressure corresponding to the given flow is applied as load on the elastic vessel which is treated as an elastic body in terms of elasticity theory. The result of the computation is then used to update the wall shape of the vessel.

New fluid nodes need to be initialized with values of the density $\rho$ and the velocity $\mathbf{u}$ based on the populations $f_{i}$ belonging to the neighboring fluid nodes. In order to determine the missing populations at node $(x, y)$, three separate cases shown in Fig. 3 can occur:

- If the neighboring nodes of $(x, y)$ in direction $i$ and opposite direction $\bar{i}$ are both solid, $f_{i}$ and $f_{\bar{i}}$ are set to their equilibrium value $f_{i}^{\text {eq }}$ and $f_{\bar{i}}^{\text {eq }}$, respectively.

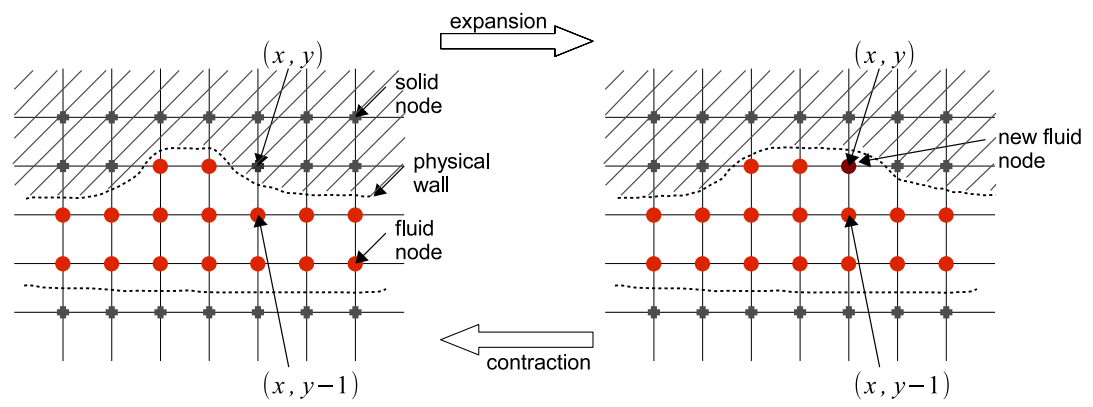

Fig. 2. (Color online) Sketch of lattice illustrating the concept of wall displacement based on node type changes. Gray crosses indicate solid nodes; red filled circles denote fluid nodes. The dark red disk designates a node that has changed its type. The virtual wall (dashed line) separates the solid and the fluid domains. 


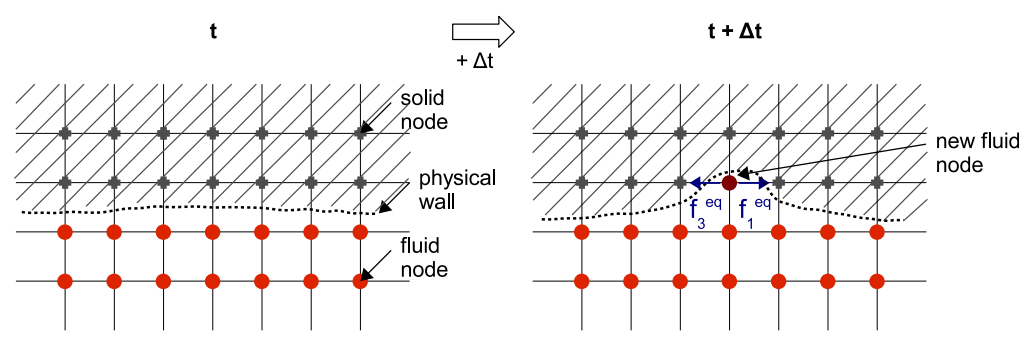

(a)
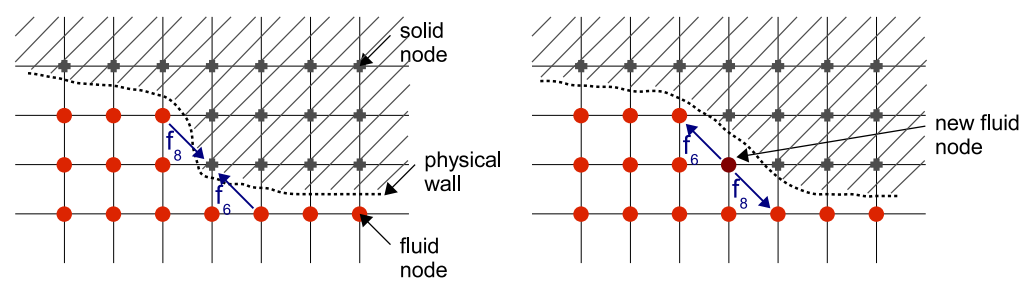

(b)
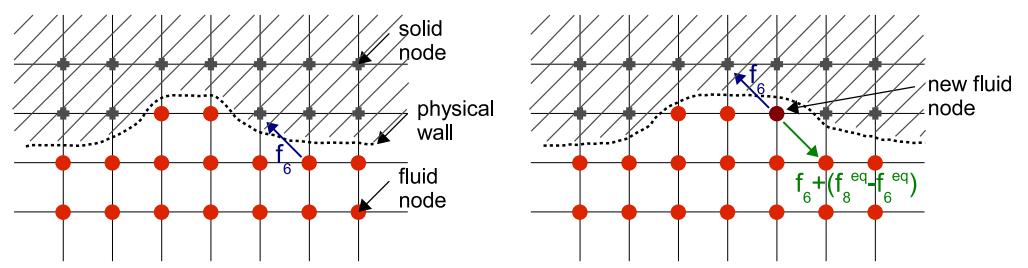

(c)

Fig. 3. (Color online) Sketch of the initialization of new fluid nodes (dark red disks) in three cases. (a) A new fluid node having solid neighbors in direction 1 and $3 . f_{1}$ and $f_{3}$ at the new fluid node are set to their equilibrium values. (b) A new fluid node having fluid neighbors in direction 6 and 8. $f_{6}$ and $f_{8}$ at the new fluid node are gained from propagation. (c) A new fluid node having a fluid neighbor in direction 8 and a solid neighbor in the opposite direction 6 . At the new fluid node, $f_{6}$ is gained from propagation and $f_{8}$ using the bounce-back rule for the nonequilibrium part.

- If the neighboring nodes of $(x, y)$ in direction $i$ and $\bar{i}$ are both fluid, $f_{i}$ and $f_{\bar{i}}$ are obtained from the LB propagation scheme.

- If the neighboring node in direction $i$ is fluid and the neighboring node in direction $\bar{i}$ is solid, $f_{\bar{i}}$ is obtained from the LB propagation step and $f_{i}$ is computed using a completion rule for the nonequilibrium part of the population, Eq. (2.10).

Mass conservation is imposed when nodes change their type, through a local rescaling procedure. It takes into account only the nearest neighbors of the node changing its state. At expansion, mass density is redistributed in the following way: let $(x, y)$ be a node changing its from solid to fluid and $\sum \rho_{n b}$ the sum of the densities at fluid nodes neighboring node $(x, y)$. After initializing the new fluid node $(x, y)$ as described above, its populations and those from the neighboring fluid 
nodes are rescaled by the factor

$$
\frac{\text { old local mass }}{\text { new local mass }}=\frac{\sum \rho_{n b}}{\sum \rho_{n b}+\rho(x, y)}<1 .
$$

Density and velocity are computed based on these new populations. Note that, being $\mathbf{u}=0$ at the new node appearing in proximity of the wall, momentum is also conserved.

Similarly, mass is redistributed locally when a fluid node changes its state from fluid to solid (contraction) and all fluid nodes surrounding the disappearing fluid node are rescaled by a scaling factor $>1$.

We remark that, compared to the method proposed in Refs. 8 and 32, where a new fluid node is initialized with the equilibrium populations, our procedure includes the nonequilibrium component which becomes preeminent in proximity of the wall. In the follow-up we will show that such a choice guarantees a continuous dynamics and robust numerical scheme in the wall region.

\subsection{Continuous wall displacement}

The continuous bounce-back boundary condition is based on combining the bounceback scheme with spatial interpolations. This method was first introduced by Bouzidi et $a l .{ }^{40}$ and later extended in Ref. 41 . The wall can be placed arbitrarily between fluid and solid nodes, i.e. at an arbitrary distance from the last fluid node in a given direction. The unknown populations are then computed by using interpolations and the bounce-back rule.

In this work, a linear interpolation scheme will be used to determine the unknown populations near the wall with directions pointing into the fluid domain. For quadratic interpolation formulas, the reader is referred to Refs. 40 and 41 . The wall is imagined to be located at a certain distance $q$ from the last fluid node (Fig. 4). In the following it will be termed virtual wall because it only represents the interface between solid and fluid domain. The lattice spacing $\Delta x$ is set to one; thus, $q$ ranges

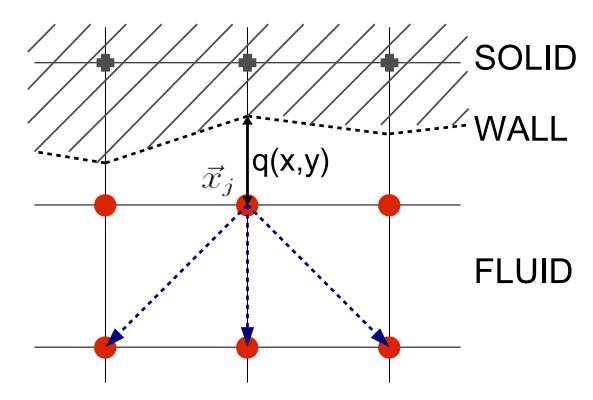

Fig. 4. (Color online) Continuous bounce-back boundary condition for a virtual wall located at distance $q$ from the last fluid node $x_{j}$. Gray crosses indicate solid nodes, red filled circles denote fluid nodes. Dashed blue arrows represent directions $i$ of unknown populations $f_{i}$ which are determined using the linear interpolation in Eqs. (3.1) or (3.2). 
between zero and one. If $q=1 / 2$, the normal bounce-back rule is recovered (halfway bounce-back on the link). Let $\mathbf{x}_{j}$ be a fluid node such that $\mathbf{x}_{s}=\mathbf{x}_{j}+\mathbf{c}_{i}$ is a solid node and let $\bar{i}$ denote the opposite direction of $i$ (thus, $\mathbf{c}_{\bar{i}}=-\mathbf{c}_{i}$ ). For the unknown populations at node $\mathbf{x}_{j}$, the following linear interpolation is used.

$$
\begin{aligned}
& f_{\bar{i}}\left(\mathbf{x}_{j}, t+1\right)=2 q f_{i}^{*}\left(\mathbf{x}_{j}, t\right)+(1-2 q) f_{i}^{*}\left(\mathbf{x}_{j}-\mathbf{c}_{i}, t\right), \quad q<\frac{1}{2}, \\
& f_{\bar{i}}\left(\mathbf{x}_{j}, t+1\right)=\frac{1}{2 q} f_{i}^{*}\left(\mathbf{x}_{j}, t\right)+\frac{(2 q-1)}{2 q} f_{\bar{i}}^{*}\left(\mathbf{x}_{j}, t\right), \quad q \geq \frac{1}{2},
\end{aligned}
$$

where the star denotes the post-collisional state. For the exact wall boundary location, the bounce-back scheme in combination with spatial interpolation ${ }^{40}$ (continuous bounce-back boundary condition) is applied to reduce discretization errors. The linear interpolation given by Eqs. (3.1) and (3.2) is used to determine the unknown populations near the wall with directions pointing into the fluid domain. It is applied to all lattice links crossing the virtual wall. The method allows a continuous displacement of the wall because the wall can be placed anywhere between fluid and solid nodes.

In the simulations, two arrays of $q$-values are used, one for the lower and one for the upper boundary. Thus, for every fluid node $\left(x, y_{b}\right)$ next to a boundary, $q_{b}(x)=$ $q\left(x, y_{b}\right)$ determines the distance from node $\left(x, y_{b}\right)$ to the wall. Here, the subscript in $y_{b}$ specifies whether the lower boundary ( $b=$ lower) or the upper boundary ( $b=$ upper) is considered. For the sake of simplicity, the subscript is omitted if not necessary to specify which boundary is considered.

The parameter $q$ of a certain node next to the boundary is linked to the pressure at this node. More details about the exact relationship between $q$ and the pressure are described further down. The approach of coupling $q$ with the pressure and using continuous bounce-back boundary conditions allows a more gradual displacement of the (virtual) wall instead of moving it by one lattice unit within a single time-step. ${ }^{42}$

Once $q_{b}$ are computed, the virtual radius $R(x)$ at a position $x$ of the channel is introduced to specify the exact distance of the wall from the centerline of the channel. It can be computed in the following way:

$$
R(x)=\frac{q_{\text {upper }}(x)+\left(y_{\text {upper }}(x)-y_{\text {lower }}(x)\right)+q_{\text {lower }}(x)}{2} .
$$

\subsection{Pressure - radius relationship}

The change of node type is dependent on the local pressure surrounding a considered node. Subject to this pressure, the wall is displaced. Transmural pressures $p_{\text {tm }}$ are assigned to each node, increasing with the distance from the vessel centerline. Nodes located further away from the centerline have a higher values of $p_{\mathrm{tm}}$, so that a higher pressure is needed for an outwards displacement of the wall, i.e. for changing the type of a node from solid to fluid. 
For simplicity, a linear relationship between the transmural pressure $p_{\text {tm }}$ and the vessel radius $R$ is assumed, similar to the one of the pulmonary blood vessels, ${ }^{39}$ reading

$$
p_{\mathrm{tm}}=p_{0}+\alpha\left(R-R_{0}\right)
$$

Here, $\alpha$ is a compliance constant (incorporating Young modulus, wall thickness and other physical properties) and $p_{0}=p\left(R_{0}\right)$ is the pressure corresponding to $R_{0}$ in equilibrium (no-flow conditions). The relation (3.4) constitutes a good approximation of wall properties for large arteries, ${ }^{23}$ with the fluid and transmural pressures trying to achieve a mechanical balance. ${ }^{10}$ In the literature, other nonlinear relations between pressure and radius are available ${ }^{13,43}$ and the present method can be easily extended to them.

In the algorithm, the transmural pressures are assigned to each node. The criterion for changing the type of a node is related to the parameter $q$ of the continuous bounce-back boundary condition. Let $p\left(x, y_{b}\right)$ denote the current pressure at a fluid node $\left(x, y_{b}\right)$ next to the wall and $p_{\mathrm{tm}}\left(x, y_{b}\right)$ the transmural pressure at this node. The difference to the transmural pressure of the neighboring node (in $y$-direction) is given by the parameter $\alpha$ in the linear pressure-radius relationship. At every time-step, the parameter $q$ is updated based on the following relationship.

$$
q\left(x, y_{b}\right)=\frac{p\left(x, y_{b}\right)-p_{\mathrm{tm}}\left(x, y_{b}\right)}{\alpha}+\chi(x, t)
$$

where the effect of the elastic wave is accounted for, by solving the wave equation for the displacement $\chi$ :

$$
\frac{\partial^{2} \chi}{\partial t^{2}}-c_{w}^{2} \frac{\partial^{2} \chi}{\partial x^{2}}=0,
$$

(cfr. Eq. (2.7)). In other words, by Eq. (3.5), $q$ changes locally in space and time due to the pressure and to the wall elastic displacement. This creates a bi-directional feedback on the wall arising from the local value of the fluid density.

The value of $q$ determines whether a node type change occurs or not. If $q\left(x, y_{b}\right)$ becomes greater than one because of a large raise of pressure, the solid node next to $\left(x, y_{b}\right)$ changes its state to fluid. On the other way around, if $q\left(x, y_{b}\right)$ falls below zero because of a large pressure drop, the node $\left(x, y_{b}\right)$ changes its state from fluid to solid.

As soon as a node type change occurs (say when $q>1$ or $q<0$ ), $q$ has to be reset to a value between zero and one. This is done as follows. If $q$ exceeds one, thus $q=1+\Delta q$ with $\Delta q>0$, the new $q$ is set to $\Delta q$ if $\Delta q<1$ and set to one if $\Delta q>1$. In a similar way, if $q$ falls below zero, thus $q=-\Delta q$ with $\Delta q>0$, the new $q$ is set to $(1-\Delta q)$ if $\Delta q<1$, and set to zero if $\Delta q>1$.

The profiles of the vessel radius for different compliances and in stationary flow conditions are illustrated in Fig. 5. The data are compared with the functional form $R(x)=\left(c_{0} x+c_{1}\right)^{1 / 5}$, with $c_{0}$ and $c_{1}$ two fitted parameters, being the solution of the one-dimensional Navier-Stokes equation in the absence of logarithmic dependence 


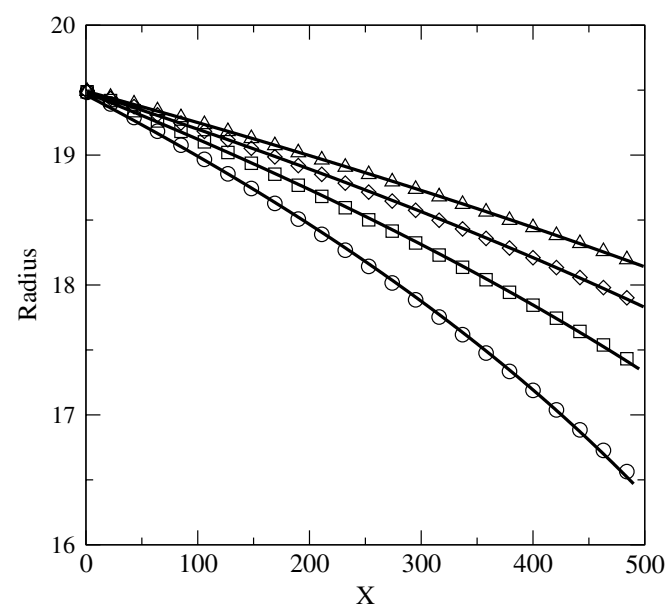

Fig. 5. Computed radius profiles compared with $R(x)=\left(c_{0} x+c_{1}\right)^{1 / 5}$, with $c_{0}$ and $c_{1}$ fitted parameters. Symbols refer to the LB data and lines to the fitted curves, with $\alpha=6$ (circles), 8 (squares), 10 (diamonds) and $12 \times 10^{-3}$ (triangles). Data are for $\nu=1 / 6$ and $\gamma=0.30$.

of the radius. ${ }^{3}$ The fitted curves show a satisfactory match with the LB profiles, lending good confidence on the accuracy of the LB scheme in steady conditions. In the following, we will move our analysis to the time-domain.

\subsection{Windkessel closure}

To overcome the problem caused by the outflow boundary condition prescribing a constant pressure, alternative boundary conditions are possible. Actually, in distributed models of blood flow, the peripheral circulation is often considered as a distal boundary condition for the arterial system. As a representation for the impedance at the end of the considered vessel, the Windkessel model is used for the description of the arterial termination and, properly validated, can avoid spurious reflections at the outlet, especially in time-dependent problems. ${ }^{44}$

In the same spirit, instead of imposing constant pressure at the outlet, we apply a two-element Windkessel model to determine the instantaneous pressure in that region. We consider a peripheral termination of the vessel described by the following pressure-flow relation ${ }^{45}$ :

$$
Q=\mathcal{C} \frac{d p}{d t}+\frac{p}{\mathcal{R}}
$$

where $Q$ is the outflow and $p$ is the arterial pressure, $\mathcal{R}$ represents the peripheral resistance and $\mathcal{C}$ the peripheral compliance. To determine the pressure $p_{\text {out }}$ at the outlet, a forward Euler method with a unit time-step is used to integrate Eq. (3.7), yielding:

$$
p_{\text {out }}^{t+1}=p_{\text {out }}^{t}+\frac{1}{\mathcal{C}}\left(Q^{t}-\frac{p_{\text {out }}^{t}}{\mathcal{R}}\right) .
$$


Since $p_{\text {out }}$ is updated at every time instant by virtue of Eq. (3.8), the radius of the outflow section changes as well, which gives a more realistic boundary condition as arising from the properties of the arterial periphery. We studied the response of the system either to a pulse, prescribed by $\rho_{\text {in }}^{\text {pulse }}=\bar{\rho}+A \exp \left[-50\left(\frac{t-t_{\text {pulse }}}{2 T}\right)^{2}\right]$, where $A=$ 0.07 and $T=10$ are the amplitude and the width of the pulse, and to a sinusoidal wave of the same amplitude $A$ and period $T$. The symbol $\bar{\rho}=1.0$ denotes the density of the fluid at rest and $t_{\text {pulse }}=10000$ is the time when the pulse reaches its maximum.

A number of test simulations have been carried out to calibrate $\mathcal{C}$ and $\mathcal{R}$ for a channel with initial dimensions $500 \times 40$ embedded in a computational domain of dimensions $500 \times 60$. The fluid viscosity is chosen to be $\nu=1 / 6$ and the compliance constant $\alpha=0.003$.

The presence of a fast sound wave with speed $c=1 / \sqrt{3}$ (corresponding to $\gamma=0$ in Eq. (2.8)) requires a fine tuning of the $\mathcal{C}$ and $\mathcal{R}$ parameters. In particular, we tried several combinations and acceptable results with negligible oscillations were achieved for $\mathcal{C}=10^{4}$ and $\mathcal{R}=0.5$. Vice versa, for a slower sound wave $(\gamma \rightarrow 1 / 3)$ we obtained a much well-behaved response, with the quality of the results being rather independent of the parameters $\mathcal{C}$ and $\mathcal{R}$.

\section{Results}

Several numerical experiments have been carried out to show the effectiveness of our approach. Readers interested in code optimization and performance of LB algorithms are referred to other authors. ${ }^{46-48}$ Although the focus is on the feasibility of the methodology, the overall algorithm is proved to be computationally efficient as in a standard LB method, since it does not explicitly account for the wall structure equations. The structure is modeled through its indirect effect on the fluid and the developed approach remains local, which makes it suitable and attractive for parallel computing.

In the simulations, the calculations are based on the LB equation with single relaxation time over the D2Q9 lattice (Fig. 1) and LB units are used if not otherwise specified, with $\operatorname{Re} \simeq 10^{-2}$.

Figure 6(a) illustrates the behavior of the channel radius once a pulse signal is injected from the inlet. The channel response to the "flicked" signal shows a faster wavefront carried by the sound wave that rapidly decays to build-up the characteristic shape. Due to the Windkessel filter, no sonic reflection exhibits at the outlet. The sound wave is soon followed by the slower elastic pulse that propagates at constant speed and shape. The computed numerical speed matches the assigned $c_{w}$.

Figure 6(b) illustrates the response of the channel radius to a sinusoidal inflow: after an initial transient, the travelling wave is clearly visible. Here again, there is no substantial sonic reflection from the wall. The evolution of the system pressure and velocity at midchannel position is shown in Fig. 7, exhibiting the nontrivial evolution of the fluid variables as the radius deforms. The continuous evolution of the latter are 


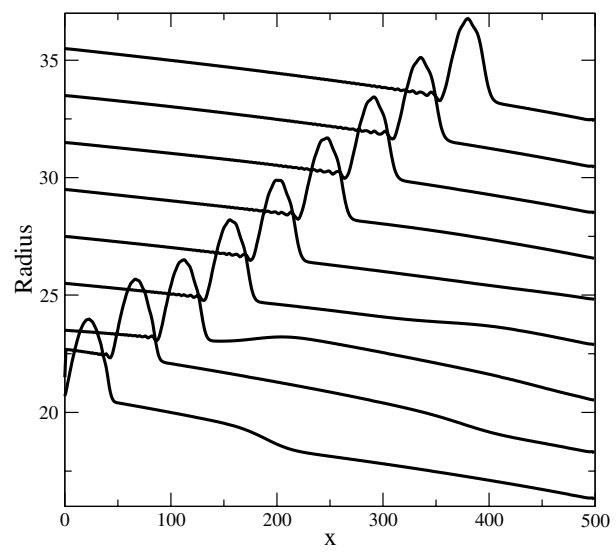

(a)

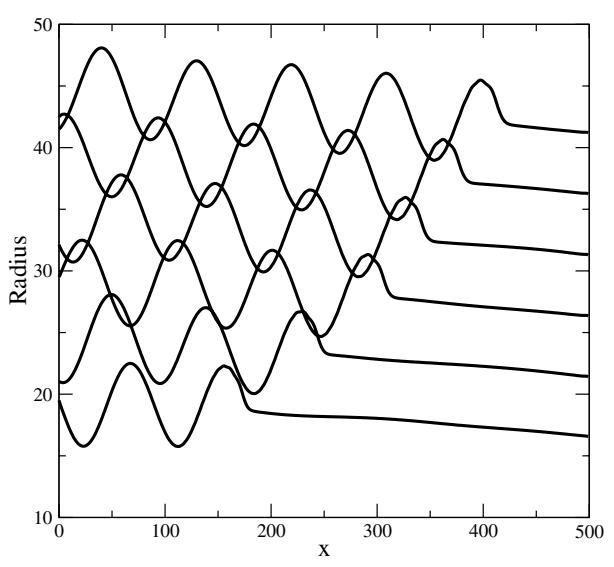

(b)

Fig. 6. Channel radius responding to the pulse (a) and sinusoidal (b) signal. Curves correspond at different time instants and have been shifted upwards by an arbitrary value for clarity. Data are for $\nu=1 / 6, \alpha=25 \times 10^{-3}, \gamma=0.30$ and $c_{w}=0.045$.

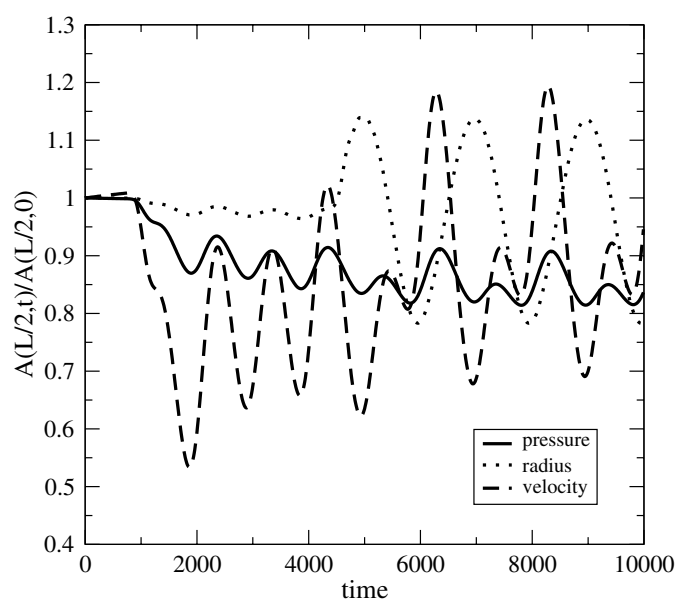

Fig. 7. Pressure (solid line), radius (dotted line) and longitudinal velocity (dashed line) at midchannel and on the centerline divided by the sinusoidal inlet signal. After a short transient, periodicity of the system becomes evident. Data are for $\nu=1 / 6, \alpha=25 \times 10^{-3}, \gamma=0.30$ and $c_{w}=0.002$.

further evaluated in proximity of the walls, in order to appreciate any discontinuity due to the mesh nodes dynamical labeling. Figure 8 displays $\rho$ and $u_{x}$ at a fluid node near the wall. In all cases, the peak values repeat at same frequency. The first peak should not be taken into consideration because expansion and contraction are allowed for $t>1000$, when fully developed flow is guaranteed.

Differently than the results with classical halfway bounce-back on the link boundary condition, the solution is free from superposed oscillations or spikes. ${ }^{27,42}$ 


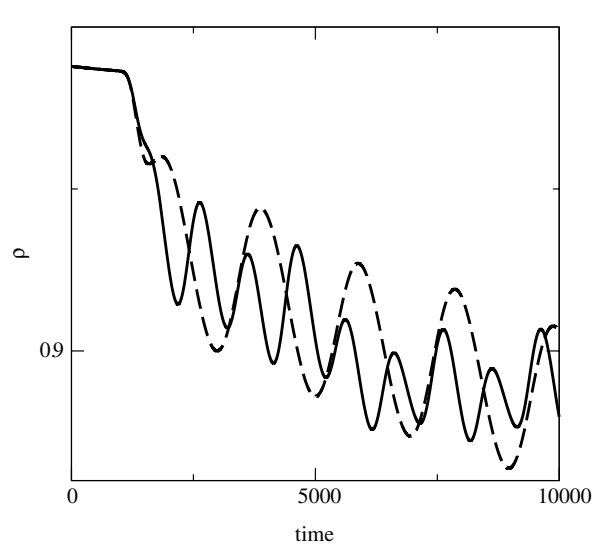

(a)

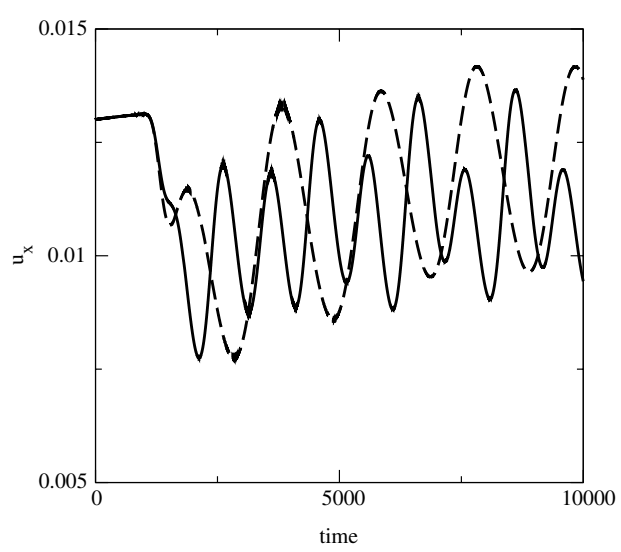

(b)

Fig. 8. Time evolution of density $\rho$ (a) and longitudinal velocity $u_{x}$ (b) at midchannel near the wall for the sinusoidal inflow signal. Data are for $\nu=1 / 6, \alpha=25 \times 10^{-3}$ and $\gamma=0.30$. Curves refer to $T=1000$ and $c_{w}=0.044$ (solid lines) and to $T=2000$ and $c_{w}=0.014$ (dashes lines).

This is due to the continuous displacement of the wall and to the fact that the LB populations are updated several times before a node change occurs.

Figure 9 reports the time evolution of the velocity field for the pulse signal. The sound wave carries an intense velocity field, and is followed by a slower elastic wave. By virtue of the local expansion, a locally smaller velocity field is visible. No unphysical oscillations appear in the time histories with an overall smooth response. Figure 10 reports the time evolution of the velocity field for the sinusoidal signal. This has been artificially magnified only for visualization purposes. Similarly to the pulse case, as the sound wave sets in, a modulated elastic wave develops, with velocity field concentrated where the vessel is more compressed as a consequence of the quasi-conserved energy.

\section{Towards Physiological Experiments: Blood Flow Through Stents}

In many vascular pathologies, when the arterial lumen is extremely reduced (stenosis), the stenting methodology is successfully employed since many decades. It is based on the implant of a tubular endoprosthesis (stent) to re-open and support the arterial wall. Despite its complex geometrical structure and a variety of mechanical characteristics, a stent can be schematically represented as a stiff cylindrical wiremesh placed in the vessel to prevent or to correct narrowing of the section. ${ }^{4}$ Although the stent implantation changes the geometry of the vessel and consequently induces important disturbances in the local flow, a relevant effect in the wallfluid interaction is the change of the compliance due to the sudden rising of the elasticity coefficient, and the features of the propagation. The presented model can easily be applied to a wall with variable elastic properties. Since the stented portion is 


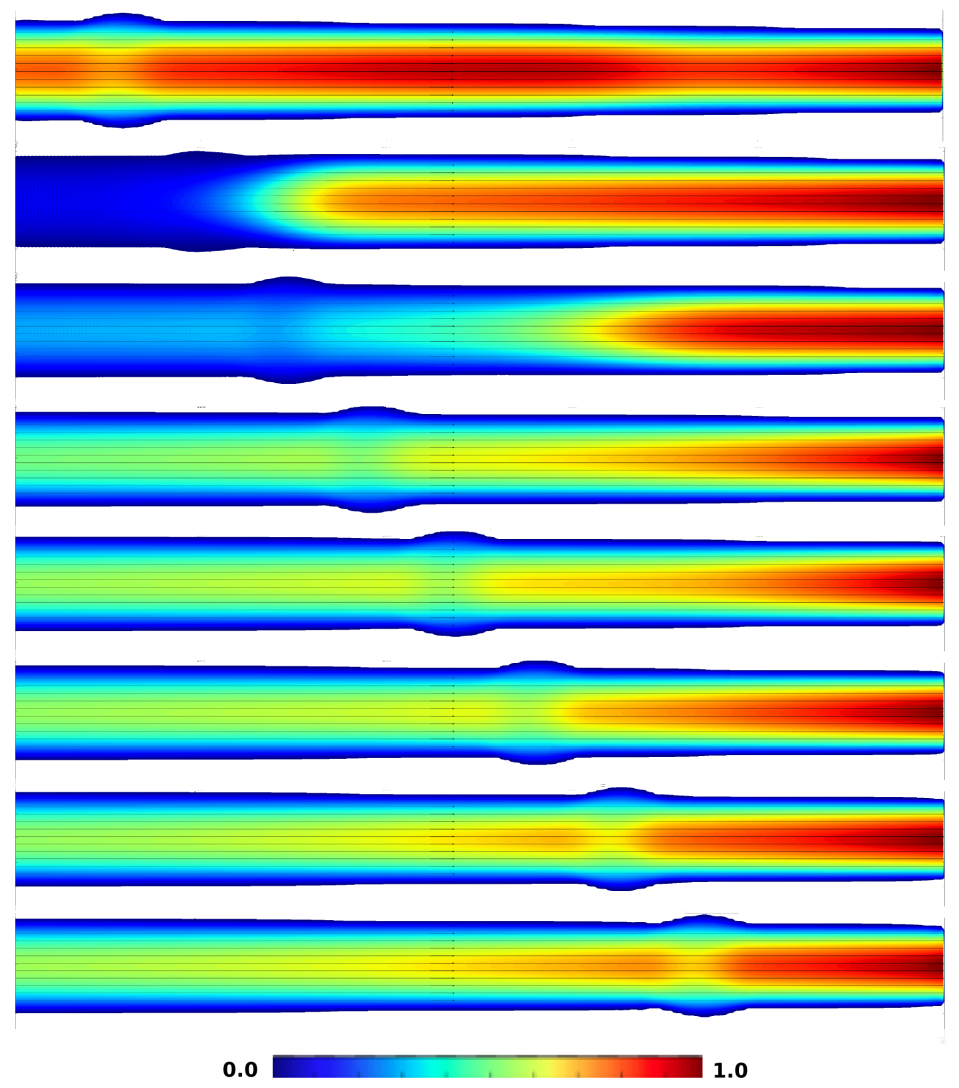

Fig. 9. (Color online) Velocity field in a compliant channel at different instants for the inlet pulse signal. The velocity field has a leading sound wave followed by a slower elastic wave, having a minimum velocity in correspondence of the channel expansion.

stiffer than the rest of the vessel, it has a higher stiffness parameter than the unstented part. Therefore, the idea to model the stent by varying the compliance parameter comes naturally. Instead of taking a constant $\alpha=\alpha_{0}$ in the pressure-radius relationship (3.4), $\alpha$ abruptly raises to $\alpha_{s}>\alpha_{0}$ in the stiffer part representing the stent. Thus, $\alpha$ varies with $x$, i.e. $\alpha=\alpha(x)$. In order to avoid a discontinuity in $\alpha(x)$ at the edges of the stent due to the considerable difference between the compliant vessel and the stiffer stented part, $\alpha$ is chosen as a rapidly continuously changing function:

$$
\alpha(x)=\alpha_{0}\left[1+\delta \exp \left(-\left(\frac{x-x^{*}}{\sigma}\right)^{8}\right)\right] .
$$

Here, $\sigma$ is the stent half-length, $x^{*}$ is the point around which the stent is centered and $\delta=\frac{\alpha_{s}-\alpha_{0}}{\alpha_{0}}$ the stiffness radius. ${ }^{49}$ For $\alpha_{s}=\alpha_{0}$, the compliance parameter of the unstented vessel is recovered. By varying the value of $\sigma$ and $\delta$ it is possible to roughly model a number of mechanical properties of stents and the local stiffening or softening of the artery. ${ }^{49}$ 


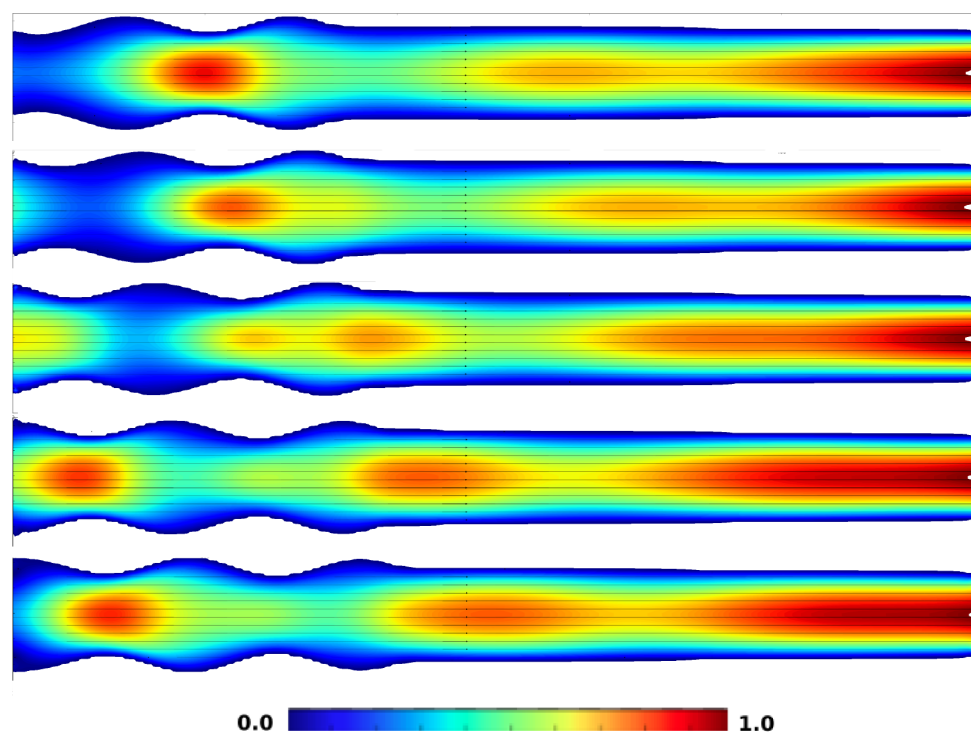

Fig. 10. (Color online) Same as Fig. 9, but with a sinusoidal inlet signal. Only the field intensity above $|u|>0.001$ is shown to appreciate the propagating wave front.

With $\alpha=\alpha(x)$ defined by Eq. (5.1), the pressure-radius relationship in Eq. (3.4) and the relationship (3.5) are easily generalized. By this, nodes corresponding to the stented part have higher transmural pressures than nodes representing the unstented vessel wall. As described above, the transmural pressures are coupled to the parameter $q$ of the continuous bounce-back boundary condition as in Eq. (3.5).

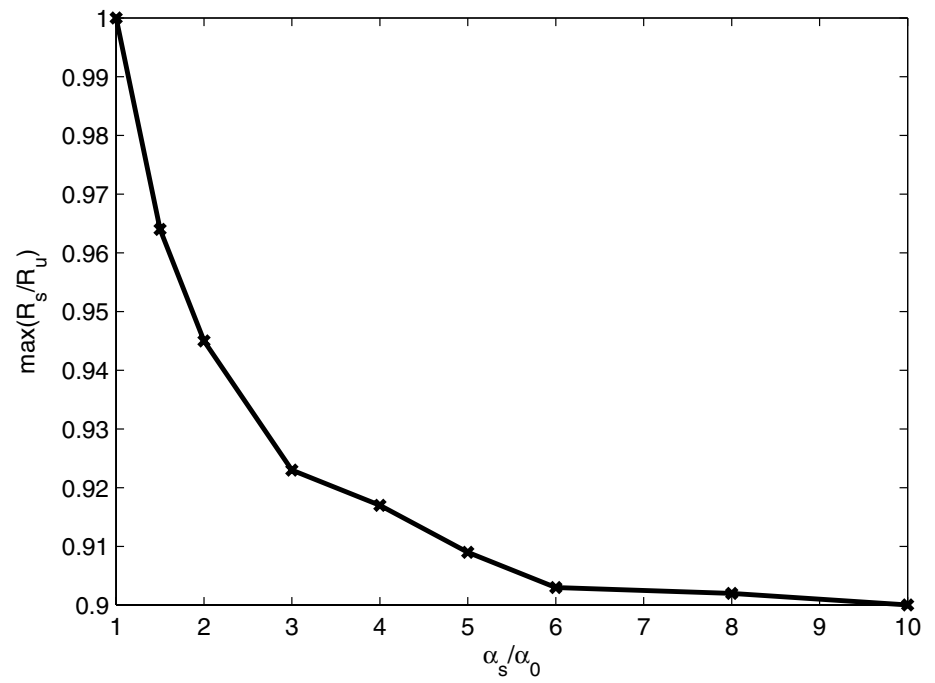

Fig. 11. Ratio between the stented and the unstented maximum deformation at the center of the stented segment $\left(\alpha_{0}=0.007\right) . R_{s}$ and $R_{u}$ denote the radius of stented and unstented vessel, respectively. 
A channel of dimensions $500 \times 40$ is considered, embedded in a computational domain of dimensions $500 \times 60$. A stent of length 100 lattice units is imagined to be located in the middle of the vessel, so $\sigma=50$ and $x^{*}=250$. The compliance parameter $\alpha_{0}$ of the unstented part has been chosen to be 0.007. An oscillating pressure is prescribed at the inlet, whereas constant pressure boundary conditions are imposed at the outlet (the coupling LBM/WK not being developed and tested in detail so far). Simulations with different $\alpha_{s}$ have been carried out and the ratio between the stented and the unstented maximum deformation at the center of the stented segment has been computed. Figure 11 depicts this ratio as a function of $\alpha_{s} / \alpha_{0}$. As expected, the ratio decreases with $\alpha_{s} / \alpha_{0}$ and tends asymptotically to the value of the rigid wall.

\section{Conclusions and Perspectives}

Summarizing, we have presented a new variant of the LB scheme which permits to handle two-dimensional hemodynamic flows with distensible walls. Elastic wave propagation along the wall, as a result of fluid-wall coupling is explicitly demonstrated, which, to the best of our knowledge, was not shown before in the framework of LB simulation.

The present approach has shown that in two dimensions the continuous bounceback method, coupled with a constitutive relation, can account for compliant walls. In addition, the propagation of a pressure wave is sustained and controlled by the compressibility of the inner fluid. Such control is nicely achieved in the framework of the current LB scheme.

The central point of the present approach stems from imposing a pressure-area constitutive relation and, at the same time, by circumventing the complication of representing explicitly the elastic wall membrane. The approach is particularly effective in the axisymmetric case, but cannot be applied "as is" in a more general situation, where geometrical complexity must be taken into account in full.

The most natural and relevant extension of the present method pertains to the treatment of three-dimensional flows, in particular for the case of irregular arterial geometries reconstructed from high-resolution tomographic data. When used in conjunction with the LB method, possibly with the further addition of flowing red cells in order to increase the physical realism of the blood representation, the approach has proven very powerful to simulate realistic clinical cases, in a matter of a few CPU hours. ${ }^{33}$ The implementation of the continuous bounce-back method to three-dimensional systems is currently under way within the MUPHY software package. ${ }^{33}$ The methodological details of the three-dimensional extension will be the object of a future publication.

\section{Acknowledgments}

This research was accomplished within the context of the project BioCompatible Materials and Applications (BCMA) initiated by the AIT Austrian Institute of 
Technology GmbH. It was partly funded by AIT and the Federal state of Lower Austria and co-financed by the EC (EFRE).

Illuminating discussions with Jonas Lätt and Dimitrios Kondaxakis are kindly acknowledged.

The Italian project Interomics is greatly acknowledged.

\section{References}

1. Eurostat, Health Statistics - Atlas on Mortality in the European Union, Office for Official Publications of the European Communities (Eurostat Commission, Luxembourg, 2009).

2. B. M. Kaess et al., J. Amer. Med. Ass. 308, 875 (2012).

3. A. Quarteroni and L. Formaggia, Handbook of Numerical Analysis 12, 3 (2004).

4. D. N. Ku, Annu. Rev. Fluid Mech. 29, 399 (1997).

5. D. M. Wootton and D. N. Ku, Annu. Rev. Biomed. Eng. 1, 299 (1999).

6. J. Alastruey, K. H. Parker, J. Peiró and S. J. Sherwin, J. Eng. Math. 64, 331 (2009).

7. F. Breitenecker, S. Wassertheurer and M. Wibmer, A one-dimensional model of blood flow: Mathematical analysis and numerical treatment. In IFMBE Proceedings (EMBEC), 2002.

8. D. Leitner, S. Wassertheurer, M. Hessinger and A. Holzinger, Elektrotech. Inf. Tech. 123, $152(2006)$.

9. B. Chopard and S. Marconi, J. Stat. Phys. 107, 23 (2002).

10. G. M. Doctors, M. D. Mazzeo and P. V. Coveney, Comput. Phys. Commun. 181, 1584 (2010).

11. G. Pontrelli, Comput. Meth. Biomech. Biomed. Eng. 7, 79 (2004).

12. G. A. Buxton, R. Verberg, D. Jasnow and A. C. Balazs, Phys. Rev. E 71, 056707 (2005).

13. N. M. Maurits, G. E. Loots and A. E. P. Veldman, J. Biomech. 40, 427 (2007).

14. M. Krafczyk, J. Tölke, E. Rank and M. Schulz, Comput. Struct. 79, 2031 (2001).

15. C. S. Peskin, J. Comput. Phys. 25, 220 (1977).

16. M. P. Rast, Int. J. Numer. Meth. Fluids 19, 1115 (1994).

17. K. Perktold and G. Rappitsch, J. Biomech. 28, 845 (1995).

18. S. Succi, The Lattice Boltzmann Equation (Oxford University Press, Oxford, 2001).

19. S. Succi, R. Benzi and F. Higuera, Physica D: Nonlinear Phenomena 47, 219 (1991).

20. S. Succi, Eur. Phys. J. B 64, 471 (2008).

21. H. Fang, Z. Lin and Z. Wang, Phys. Rev. E 57, 25 (1998).

22. H. Fang, Z. Wang, Z. Lin and M. Liu, Phys. Rev. E 65, 51925 (2002).

23. A. G. Hoekstra, J. van't Hoff, A. M. Artoli and P. M. A. Sloot, Future Gener. Comput. Syst. 20, 917 (2004).

24. A. J. C. Ladd, J. Fluid Mech. 271, 285 (1994).

25. A. J. C. Ladd, J. Fluid Mech. 271, 311 (1994).

26. H. Li, X. Lu, H. Fang and Y. Qian, Phys. Rev. E 70, 026701 (2004).

27. X. Descovich, Lattice Boltzmann modeling and simulation of incompressible flows in distensible tubes for applications in hemodynamics, Ph.D. thesis, Vienna University of Technology (2012).

28. P. W. Serruys, M. J. B. Kutryk and A. T. L. Ong, New Eng. J. Med. 354, 483 (2006).

29. H. C. Lowe, S. N. Oesterle and L. M. Khachigian, J. Amer. Coll. Cardiol. 39, 183 (2002).

30. D. A. Wolf-Gladrow, Lattice-Gas Cellular Automata and Lattice Boltzmann Models: An Introduction (Springer, 2000).

31. R. Benzi, S. Succi and M. Vergassola, Phys. Rep. 222, 145 (1992).

32. D. Leitner, S. Wassertheurer, M. Hessinger, A. Holzinger and F. Breitenecker, Lect. Notes Comput. Sci. 4799, 213 (2007). 
33. S. Melchionna, M. Bernaschi, S. Succi, E. Kaxiras, F. J. Rybicki, D. Mitsouras, A. U. Coskun and C. L. Feldman, Comput. Phys. Commun. 181, 462 (2010).

34. G. Pontrelli, S. Ubertini and S. Succi, J. Stat. Mech. Theory and Experiment 6, P06005 (2009).

35. C. R. Ethier and C. A. Simmons, Introductory Biomechanics: From Cells to Organisms (Cambridge University Press, 2007).

36. S. Marconi, B. Chopard and J. Latt, Int. J. Mod. Phys. C 14, 1015 (2003).

37. J. M. Buick and J. A. Cosgrove, J. Phys. A, Math. Gen. 39, 13807 (2006).

38. Q. Zou and X. He, Phys. Fluids 9, 1591 (1997).

39. Y. C. Fung, Biomechanics: Circulation (Springer-Verlag, New York, 1997).

40. M. Bouzidi, M. Firdaouss and P. Lallemand, Phys. Fluids 13, 3452 (2001).

41. P. Lallemand and L. S. Luo, J. Comput. Phys. 184, 406 (2003).

42. X. Descovich, G. Pontrelli, S. Melchionna, S. Succi and M. Bammer, Modelling elastic walls in lattice Boltzmann simulations for applications in haemodynamics, in Proc. The Int. Workshop on Applied Modeling \& Simulation, Rome, September 24-27 (2012).

43. Y. C. Fung, Biomechanics: Mechanical Properties of Living Tissues (Springer-Verlag, New York, 1993).

44. N. Stergiopulos, B. E. Westerhof and N. Westerhof, Am. J. Physiol. Heart Circ. Physiol. 276, H81 (1999).

45. O. Frank, Zeitung für Biologie 37, 483 (1899).

46. R. Argentini, A. F. Bakker and C. P. Lowe, Future Gener. Comput. Syst. 20, 973 (2004).

47. M. D. Mazzeo and P. V. Coveney, Comput. Phys. Commun. 178, 894 (2008).

48. G. Wellein, T. Zeiser, G. Hager and S. Donath, Comput. Fluids 35, 910 (2006).

49. G. Pontrelli and E. Rossoni, Int. J. Numer. Meth. Fluids 43, 651 (2003). 